\title{
Gestionando entornos sociotécnicos complejos: la gobernanza del riesgo en las nanotecnologías
}

\author{
Anna Garcia Hom* \\ Ramon J. Moles Plaza*
}

\begin{abstract}
REsumen: En el campo de la generación de prospectiva, la gobernanza de riesgos sociotécnicos deviene altamente relevante como elemento estratégico de desarrollo e innovación de las organizaciones, instituciones y empresas. Siendo el riesgo un elemento central en las llamadas tecnologías emergentes, como las nanotecnologías, su gobernanza anticipatoria, es decir, su gestión anticipatoria, puede devenir un factor de clara ventaja en procesos de desarrollo tecnológico. Una visión estratégica del desarrollo e introducción de tecnologías emergentes en entornos sociotécnicos complejos nos debería permitir localizar la fase más adecuada para una intervención transdisciplinar, siendo ésta, a partir de nuestras experiencias, la fase media del proceso (midstream). Del mismo modo, en el desarrollo de los procesos de gobernanza del riesgo, deviene imprescindible el uso de recursos de autorregulación o soft law que permitan superar las rigideces normativas propias de los modelos de regulación clásica o hard law. Ello puede permitir optimizar las inversiones y la tasa de retorno en la ejecución de planes de negocio basados en la difusión social de tecnologías emergentes.

PalABRAS Clave: Gobernanza anticipatoria, nanotecnologías, entornos sociotécnicos, autorregulación.
\end{abstract}

АвSTRACT: In the field of prospective generation, governance of sociotechnical risks becomes highly relevant, as a strategic elementof development and innovation of organizations, institutions and companies. Risk is a central element in the known as emerging technologies such as nanotechnology; hence its anticipatory governance or its anticipatory management can become a factor of clear advantage in technological processes. A strategic vision for the development and introduction of emerging technologies in complex sociotechnical environments should allow us to locate the most appropriate stage for trans-disciplinary intervention, and this is, from our experience, the middle phase of the process (midstream).

Similarly, in the development of risk governance processes becomes essential to use self-regulatory resources or "soft law" to overcome regulatory rigidities typical of classical regulatory models or "hard law". This investment can be optimized and the rate of return in the execution of business plans based on the social diffusion of emerging technologies.

KEY wORDS: Anticipatory governance, nanotechnologies, sociotechnical environment, self-regulation.

\section{A MODO DE INTRODUCCIÓN: SURFEANDO LOS CONCEPTOS}

La propuesta que estructura el presente artículo se centra en incorporar el factor de la gobernanza del riesgo al debate sobre prospectiva e inteligencia competitiva a partir de la convicción de que ello puede contribuir a una mejora del posicionamiento de las organizaciones implicadas en la gestión y explotación de tecnologías emergentes en contextos sociotécnicos complejos. Concretamente, utilizaremos el caso pertinente, por su elevado carácter estratégico, de las nanotecnologías.

\footnotetext{
* Centre de Recerca en Governança del Risc, Universitat Autònoma de Barcelona. <anna.garciac@uab.cat>. <ramon.moles@uab.cat>.<www.grisc.cat/es>.+34935817122+34935817161.
} 
El desarrollo de entornos sociotécnicos complejos ha facilitado a los individuos la posibilidad de desarrollar nuevas capacidades para su interacción con aquéllos. Si bien esos entornos caracterizados en parte por la incertidumbre científica que rodean algunas de sus aplicaciones han dotado de consecuencias insospechadas sus resultados, lo cierto es que, a menudo, se asocian a percepciones de riesgos construidos socialmente. Siendo ello así, es obvio que una aproximación unidisciplinar al problema nos limitaría sobremanera nuestro conocimiento a propósito de las estrategias existentes para su gestión. Es por ello, que deberíamos favorecer nuevos modelos de análisis del fenómeno que permitan comprenderlo desde la multidisciplinariedad —en la medida en que no es posible un abordaje unilateral de la cuestión-, como tampoco lo es el propio objeto de estudio. Así, el presente artículo aboga por un abordaje multidisciplinar del fenómeno de la potencial construcción social de riesgos asociados a las nanotecnologías a la vez que formulamos un modelo de corte anticipatorio para la gobernanza en materia de riesgos potenciales y de incertidumbre científica.

Este modelo de naturaleza anticipatoria difiere y a la vez se complementa con el que podemos obtener a partir de metodologías como la prospectiva, la vigilancia tecnológica o la inteligencia competitiva, siendo que el modelo de gobernanza anticipatoria reúne dos características propias: una, ser una metodología no meramente descriptiva sino también para la intervención práctica, y, dos, implicar no sólo al sector privado sino también al sector público y a la sociedad civil. Factores, por otro lado, imprescindibles para introducir en el tejido social las llamadas tecnologías emergentes, tales como las nanotecnologías.

Para ello, y atendiendo a la definición clásica que Naciones Unidas utiliza para referirse a la gobernanza, ${ }^{1}$ en nuestro caso la aplicaremos al campo de los riesgos sociotécnicos aplicados a las nanotecnologías, si bien y aunque atendiendo a las mismas consideraciones del triple ejercicio de autoridad política, económica y administrativa, contemplaremos tres stakeholders básicos a tener en cuenta: el sector público, el privado y la sociedad civil. Conocer y actuar en las interrelaciones que se desarrollan en este triángulo puede resultar altamente estratégico para las organizaciones, instituciones y centros de investigación implicadas en este campo. Desde esta perspectiva, la gobernanza del riesgo definiría el ejercicio de la autoridad política, económica y administrativa para gestionar los riesgos (sociotécnicos) a todos los niveles descritos.

Es así que la gobernanza del riesgo se aparece como algo muy distinto de los peligros y de su prevención, en la medida en que, a diferencia de aquellos, los peligros se constituyen a partir de anticipaciones mentales de daños que, por sí mismas, son identificables, mesurables, cuantificables y, por tanto, gestionables (García, 2012). Ello nos situaría en otro ámbito que no es objeto de este trabajo: el de la prevención de peligros. En este contexto, la definición de instrumentos de gestión de peligros de prevención - reúne dificultades distintas de las derivadas de la gobernanza de riesgos; siendo que mientras los peligros — como señalábamos— son identificables, los riesgos son construidos socialmente; si los primeros son mesurables, los segundos lo son con extrema dificultad; tampoco son fácilmente cuantificables y, en suma, su gestión debería ubicarse en el contexto de la gobernanza en la medida en que su

Ejercicio de la autoridad política, económica y administrativa para gestionar un país a todos los niveles. Informe de desarrollo humano, 2007. <http://hdr.undp.org/es/informes/mundial/idh1997/capitulos/espanol/> (último acceso abril 2013). 
gestión requiere incardinarse íntimamente en lo propiamente social. En otras palabras, la introducción de tecnologías emergentes en el tejido social no puede obviar metodologías e intervenciones propias de las ciencias sociales si pretenden evitar la lotería del conflicto con y el previsible riesgo evidente de su rechazo social y el consecuente fracaso, no sólo económico por la inversión efectuada sino también organizativo, cultural y de posicionamiento en el mercado. Las nanotecnologías, entonces, se moverían en esta tesitura descrita: un contexto caracterizado por las incertidumbres y también por las ignorancias que rodean un estadio de precocidad tecnológica y, por ende, económica, social, política, jurídica y ética. Además y dada la inherente complejidad de las interacciones entre la(s) tecnología(s) y la sociedad(es)se hace aún más difícil prever con exactitud los cambios que dichas tecnologías pueden ocasionar en el tejido social en el cual se introduzcan y desarrollen. Ello se agrava en la medida en que los recursos de la regulación clásica constriñen la capacidad de los stakeholders para determinar los mecanismos eficientes que integran la gobernanza del riesgo. Frente a ello, y como veremos, los elementos autorregulatorios o de soft law suponen una alternativa factible para replantear, por ejemplo, la definición de legitimado o de procedimiento del proceso.

\section{DÓNDE: EN LA COMPLEJIDAD DE LOS ENTORNOS SOCIOTÉCNICOS}

La complejidad de los avances científicotecnológicos se manifiesta hoy en entornos en los que confluyen relaciones sociales y tecnologías diversas que se abocan a la manifestación de variados riesgos derivados del uso e implementación de aquéllos (García, 2005). A esto deberíamos añadir el elevado grado de incertidumbre organizativa, económica y regulatoria que acompaña a estos procesos en un contexto postnormal (Funtowicz y Ravetz, 1993).

La gestión de los potenciales riesgos generados en ámbitos de este tipo implica, además, el manejo de conceptos y recursos sociales en entornos de incertidumbre también científica. Es así como nos hallamos ante una realidad dibujada, de un lado, por la complejidad de los riesgos, y de otro, por las limitaciones del conocimiento anticipativo científico, siendo ambas piezas esenciales de lo que denominamos la configuración colectiva de los riesgos. En este sentido, la gestión de los riesgos tecnológicos complejos se presenta en tanto que problema no sólo científicotécnico sino también sociotécnico.

Sin embargo, aunque este fenómeno se puede manifestar en cualquier entorno sociotécnico su presencia es más evidente en los que se configuran alrededor de tecnologías emergentes, esto es, aquellas tecnologías de carácter novedoso que se hallan en el umbral de su introducción en el tejido social y respecto de las cuales se halla en ciernes su uso masivo: el caso de las nanotecnologías brilla por su presencia. Evidentemente, este fenómeno viene a complicar en gran manera la explotación de tecnologías emergentes con consecuencias evidentes para el cálculo del retorno de la inversión y la generación de beneficios.

Y es que cualquier novedad tecnológica, y más si es emergente —en el sentido anteriormente descrito - lleva asociado un conjunto de interrogantes a propósito de la rigurosidad o precisión científica en determinar los efectos (perjudiciales o no) a ella vinculada. Así, siguiendo la clasificación de Klinke y Renn (2001), en cuanto a los efectos perjudiciales, podemos clasificar los riesgos en una tipología de seis clases a 
partir de nombres de la mitología griega, (Damocles, Cíclope, Pithya, Pandora, Cassandra y Medusa) fundamentada en ocho criterios (daño potencial, probabilidad de ocurrencia, incertidumbre, ubicuidad, persistencia, reversibilidad, efecto de demora y potencial de movilización), a partir de los cuales desarrollar un concepto para la gestión del riesgo integral acentuando los mecanismos estructurales y de procedimiento así como las consideraciones orientadas a la precaución.

La sociología del conocimiento científico, por su cuenta, ha puesto de manifiesto las limitaciones del conocimiento actual en la determinación de las consecuencias de ciertos riesgos tecnológicos. En otras palabras, la gestión de riesgos sociotécnicos complejos que se centra en la gestión de riesgos involuntarios, manufacturados y resultantes de los nuevos avances tecnológicos (Hutter, 2007) incide directamente en la cuenta de resultados de las organizaciones que pretenden rentabilizar aquellas tecnologías.

Los riesgos vinculados con las tecnologías emergentes como las nanotecnologías son, más específicamente, un ejemplo del modo como con el advenimiento de una modernidad opuesta a aquello natural y tradicional, ${ }^{2}$ la ansiedad por el cambio y por el consumo de nuevas tecnologías está teniendo un conjunto de efectos susceptibles de escaparse del control humano (Burgess, 2004). De manera acorde con los planteamientos de Giddens, la mayoría de reacciones receptivas al riesgo se derivan de un sentimiento palpable de pérdida de control en un mundo fugitivo perfilado por las decisiones remotas de los sistemas de expertos corporativos (Giddens, 1991: citado en Burgess, 2004).En este marco, las fuerzas locales - esto es, las protestas de los individuos afectados - aparecen a modo de reacción ante el abrumador peso de la posición de las fuerzas globales - los agentes establecedores del riesgo- - Ante la incapacidad de hacer frente a determinados fenómenos, la única posibilidad para los individuos y para las comunidades es reaccionar de manera defensiva tratando de limitar el impacto de las amenazas tecnológicas en el seno, por ejemplo, de su localidad.

De la misma manera que el control de la incertidumbre por parte de los científicos deviene un elemento central no resuelto en los riesgos emergentes, su evaluación se sitúa también en un contexto de conocimiento no completo. A resultas de ello, como señala Stilgoe (2007: 48): "poco sentido tiene apoyarse en las recomendaciones de los científicos y de los expertos si éstos están plagados con las mismas incertidumbres que preocupan al público y a los políticos".

Entre la gran diversidad de disciplinas que han desarrollado investigación en relación con los riesgos asociados a tecnologías emergentes (sociología, psicología social, derecho, economía, prospectiva, epidemiología, entre otras) se constata en general que el núcleo central del problema seguramente resida en el hecho de que no es posible negar la posibilidad de un daño futuro pero, al mismo tiempo, tampoco disponemos de una evidencia clara de su existencia. Tal y como se desprende de la enorme controversia científica, en cualquier evento los riesgos derivados de las tecnologías

\footnotetext{
Mientras que algunas de las ventajas de la modernidad — como los teléfonos móviles — son aplaudidas, existe una asunción generalizada de que hay que pagar un elevado precio ante el rápido avance de la modernidad. Se puede afirmar que, en términos generales, los miedos asociados con algunos aspectos de la modernidad han tenido un rol central en la disminución de la confianza en la salud y en la consideración de que la industrialización ha dejado a los seres humanos desprovistos de la energía natural que fue provista por la Madre Tierra (Park, 2000: 58). En consecuencia, la creencia en el hecho de que un retorno a la naturaleza ofrece una protección contra los riesgos tiene una enorme fuerza de penetración.
} 
emergentes constituyen más una idea de lo futuro - edificada sobre nuestros pensamientos, percepciones e ideología- que no una realidad, hasta la fecha, absolutamente demostrable.

De nuevo, desde la sociología del conocimiento científico, resulta evidente que la aproximación al análisis del riesgo a menudo se ha fundamentado en un lenguaje científico y político que acostumbra ser "epistemológicamente realista, positivista, tecnológico y cognitivista” (Szerszynski et al., 1996). En el caso específico de las nanotecnologías, a este lenguaje se le añade un marco de incertidumbre que resulta de la incapacidad de satisfacer, aún, el acuerdo en cuanto a la determinación de sus efectos, esto es, acerca de sus peligros. A pesar de la persistencia del interés social por establecer instrumentos de medida de carácter uniforme, los métodos empleados para descubrir la "verdad" científica presentan divergencias considerables. Tal y como afirman Krimsky y Golding (1992: 361): "los criterios para determinar la verdad y la objetividad en la ciencia no son ni mucho menos uniformes, sino que, en su concreción, a menudo intervienen otro tipo de variables".

$\mathrm{Al}$ estado de incertidumbre antes apuntado se le suma lo que Wynne (1992) define como estado de ignorancia - en el que ignoramos no únicamente el valor que toman ciertas magnitudes y sus probabilidades, sino también qué magnitudes o eventos son relevantes en el sistema de actividad-y de indeterminación — haciendo referencia a la falta de conclusividad de un conjunto de datos o tradición-.

Esta breve descripción nos permite entender la compleja naturaleza de los entornos sociotécnicos en la medida en que desde ellos y con ellos deberíamos ser capaces de ubicar y caracterizar el espacio tiempo de las nanotecnologías.

\section{Cómo: MEDIANTE LA GOBERNANZA DEL RIESGO}

En paralelo, y aún más allá de los elementos de una buena gobernanza, a saber, participación amplia, cumplimiento imparcial de las normas, transparencia informativa, responsabilidad social, consenso en los objetivos, equidad, eficiencia, eficacia y visión estratégica, por citar algunas, ésta última es, para los propósitos de este trabajo, una herramienta imprescindible en los procesos de elaboración y toma de decisiones sobre tecnologías emergentes como las nanotecnologías en entornos sociotécnicos complejos.

Siguiendo a Fisher, Mitcham y Mahajan (2006) que propusieron un modelo de los procesos de gobernanza de la investigación científica en forma de corriente(s), proponemos caracterizar los procesos de toma de decisiones en contextos sociotécnicos complejos de modo similar. Como si del curso de un río se tratara, se distinguen tres momentos: un tramo superior (upstream), caracterizado por incluir elementos propios de procesos de investigación básica y desarrollos tecnológicos embrionarios o iniciales; un tramo medio (midstream), caracterizado a partir de elementos propios de procesos de investigación básica-aplicada, y, finalmente, un tramo inferior downstream), caracterizado por procesos de investigación aplicada y de transferencia de tecnología al mercado. Cada uno de estos tramos estaría dotado, a su vez, de un contenido relevante para sus propósitos: el primero correspondiente a la decisión de desarrollo científico de carácter básico, a menudo financiada con fondos públicos debido a la inconcreción de específicos objetivos tecnológicos que puedan ser explotables directamente; el segundo vinculado con la investigación propiamente dicha: y, el 
tercero, visualizando claramente la cristalización de intereses en relación con las tecnologías emergentes.

Cada una de estas fases conllevaría un nivel distinto de información y una capacidad de decisión determinada. De este modo, si bien en la fase inicial (upstream) el gestor del proceso dispondría de todo el poder de decisión en relación con la estrategia de investigación, objeto, metodología o financiación, por ejemplo, desconocería, en cambio, casi totalmente el tipo y la forma de la información a divulgar y el modo y el nivel de información a diseminar (en relación con su impacto social más general), sencillamente porque es también desconocida para él mismo.

El tramo inferior (downstream), en cambio, se caracterizaría, precisamente, de modo inverso: el nivel de información respecto a su impacto sería muy elevado, mientras que el poder de decisión respecto al proceso sería prácticamente nulo como consecuencia de la cristalización de intereses alrededor de las tecnologías a las que se haga referencia.

De modo distinto a los tramos superior e inferior del proceso es en el tramo medio donde tanto el nivel de información como el poder de decisión restarían a la par. Es en este tramo donde la experiencia práctica nos demostraría que hay que intervenir tratando de anticiparse a consecuencias negativas para las organizaciones que se suelen plasmar en la fase posterior (downstream). Esta anticipación se mostraría como necesaria en tanto cuanto esta fase posterior se correspondería con un estadio de cristalización de intereses que dificultaría en grado sumo cualquier intervención que, recordemos, debería ser transdisciplinar — en la medida en que sólo desde esta perspectiva plural es posible abordar la complejidad psicológica, sociológica, económica o regulatoria, por ejemplo, que abarca cualquiera de estos supuestos-.

Por otro lado, esta intervención en el tramo intermedio debería permitir también anticiparse (de ahí también la "gobernanza anticipatoria") a movimientos futuros de competidores en mercados, como éstos, altamente competitivos. En otras palabras, apuntamos a mecanismos de intervención pluridisciplinar que sumados a sistemas de vigilancia tecnológica o de inteligencia competitiva puedan permitir de un lado, a las administraciones, gobernar riesgos; del otro, a la sociedad civil, superar la construcción conflictiva de riesgos en tecnologías emergentes y, finalmente, al sector privado implicado en ellos, alcanzar posicionamientos altamente ventajosos en sus mercados. Se trataría, en resumen, de un triángulo virtuoso en que todos ganarían, que sustituiría los ya conocidos supuestos de paralización tecnológica, en que todos pierden, consecuencia de la aplicación abusiva del "principio de precaución", de carácter paralizante y que aboca a los agentes económicos a desplazar sus inversiones a mercados en que éste no se aplica en una suerte de "dumping tecnológico".

De la enorme ventaja que ofrecen estos instrumentos de gobernanza anticipatoria dan fe algunas de intervenciones realizadas en otras áreas, como la telefonía móvil, en concreto, al despliegue de las infraestructuras necesarias para su correcto funcionamiento, en que, a pesar de ubicarse ya en el tramo inferior de los relatados (downstream), ha sido posible obtener resultados positivos en dicho ámbito, o los procesos de desarrollo para el emplazamiento de un almacén centralizado de residuos radioactivos. $^{3}$

\footnotetext{
3 Las investigaciones referenciadas corresponden a algunos de los trabajos que se desarrollan en el Centro de Investigación en Gobernanza del Riesgo <www.grisc.cat/es>.
} 
Existen también ámbitos en los que es posible aplicar estas metodologías todavía en estadios intermedios como consecuencia de su "emergencia" en el contexto social: las nanotecnologías sería nuestro caso, pero al lado de ellas existen otras como las energías renovables (parques eólicos o biomasa) o grandes infraestructuras de obra pública, entre otras, las cuales representan sólo algunos de los campos en los que nuestras investigaciones nos conducen a pensar que la implantación de mecanismos de gobernanza anticipatoria en entornos sociotécnicos complejos basados en ciencias aplicadas y sociales puede aportar elementos de elevada rentabilidad política, económica y social.

\section{QUÉ: LA AUTORREGULACIÓN EN LA GOBERNANZA DEL RIESGO}

La gobernanza del riesgo requiere de unas reglas y procedimientos que pauten los procesos de generación de consenso. El carácter regulable o autorregulable de las reglas que configuran los procesos de gobernanza del riesgo depende de la arquitectura de éstos; entendiendo por arquitectura su propio diseño, esto es, la especificación de criterios de acceso al proceso, de identificación de stakeholders y de gestión de datos que permitan un acceso, más o menos general o restringido, al mismo. Mediante la arquitectura, el código regulatorio, podemos definir cómo será el proceso. Se trata, ni más ni menos, que de una decisión política cómo indica Lessig (2000), quien ha contribuido decisivamente al desarrollo del concepto de código regulatorio, que alcanza, por ejemplo, a las principales estructuras de control del proceso: las relativas al funcionamiento de los mercados, a la identificación de los stakeholders y sus intereses, a la autorización, supervisión o inspección de actividades, al establecimiento de procedimientos sancionatorios, a las normas de transparencia y a las de responsabilidad social o medioambiental. Sin embargo, estas estructuras de control pueden originarse en un contexto normativo (esto es, con supervisión del legislativo, acción del ejecutivo y supervisión judicial) público (mediante publicación en Diario Oficial), o bien en un contexto privado, generado en un organismo de normalización sin supervisión legislativa, ejecutiva ni judicial, aunque con el amplio consenso de los actores implicados y la fuerza de imponer derivada de éste que genera la autorregulación o soft law.

Estas estructuras son de gran plasticidad, es decir, es posible modelar su uso, extensión e intensidad. Son, además, estructuras al alcance tanto de la administración pública como del sector privado e incluso combinables entre ellas: existe "autorregulación regulada”, esto es, fenómenos de autorregulación incorporados por las normas del ordenamiento jurídico (así, por ejemplo, la exigencia de conformidad a normas ISO en procesos de licitación pública). La paradoja reside en lo que nos ocupa: en las limitaciones que presentan los mecanismos regulatorios en procesos de negociación de intereses de los stakeholders mediante la gobernanza del riesgo. Dicho de otro modo: si las normas jurídicas son obligatorias y su incumplimiento acarrea sanción, no podemos considerarlas un elemento negociable en los procesos de gobernanza del riesgo. Ello nos lleva a postular la necesidad imperiosa de diseñar y adaptar procesos autorregulatorios a estos ámbitos de gobernanza del riesgo en la medida en que el marco regulatorio viene dado ex ante y no es negociable ni disponible sino en sede parlamentaria o mediante procesos de revisión normativa al amparo del derecho administrativo. 
Veamos, por ejemplo, el supuesto de la legitimación de los interesados. Siendo como es en el derecho administrativo un mecanismo que vincula el derecho o interés legítimo con la legitimación en el procedimiento con vistas a participar en el mismo, en el supuesto de la gobernanza del riesgo los gestores del proceso han de ser capaces de arbitrar mecanismos de participación capaces de "dar voz a los sin voz", esto es, de conseguir incluso la participación de aquellos que en sede administrativa pudieran quedar excluidos a pesar de la relevancia del interés que pueden personificar para el éxito del proceso.

Otro ejemplo: siendo que el procedimiento administrativo se construye de un modo tasado para vertebrar la gestión de derechos e intereses legítimos, aunque siempre expresos, la gobernanza del riesgo puede permitirnos - y ahí radica el reto- vertebrar la gestión "abierta", no tasada, de intereses no tan "expresos", que fluyen bajo los conductos legales y con frecuencia bloquean los procesos jurídicos construidos con luz y taquígrafos aunque con nula sensibilidad por aquellos intereses "presuntos". Es así como llegamos a la convicción de que la gestión regulatoria del riesgo tecnológico se halla lastrada por la dificultad de aflorar el consenso social real y necesario sobre la gobernanza del riesgo mismo. Y ello porque el consenso, de haberlo, habitó genéricamente en su momento inicial en sede parlamentaria y jamás fue revisitado; resultando de este modo una pieza de museo desacorde con el tiempo de la gestión del concreto conflicto. Deberemos pues explorar nuevos territorios: la autorregulación puede ser uno de ellos.

La autorregulación se basa en normas técnicas que son estándares abiertos no obligatorios elaborados por organismos privados reconocidos a tal fin (como, por ejemplo, la ISO, AENOR o AFNOR en el caso de normas técnicas industriales o de procesos, o una asociación de periodistas o un Colegio de abogados para un código deontológico, o una asociación empresarial para una norma de responsabilidad social corporativa). Se trata pues de normas técnicas, de uso abierto, de código abierto, y ha sido precisamente su carácter abierto el que ha permitido su rápido crecimiento. La norma técnica es, en su acepción genérica, una especificación técnica internacional, comunitaria o nacional, aprobada por un organismo reconocido en materia de actividades normativas, el respeto a la cual es puramente facultativo.

La norma técnica no es, por tanto, una norma jurídica, sino el producto de una actividad técnica, sin vocación alguna, en principio ni per se, de relevancia jurídica ni de imposición obligatoria. Las normas técnicas arraigan además en la experiencia, son objeto de consenso y están destinadas a una aplicación repetitiva. Las normas técnicas se distinguen de las reglamentaciones técnicas — de carácter jurídico- en la medida en que aquellas son voluntarias y éstas obligatorias.

Hasta el momento no es frecuente, probablemente no exista ningún caso, en que la norma técnica incluya también mecanismos para la gobernanza de los riesgos asociados al desarrollo de la tecnología que la misma norma técnica describe. Al menos, no descartemos de entrada considerar el supuesto. ¿Por qué excluir de la norma técnica el análisis de los procesos de gobernanza del riesgo que puedan permitir establecer un marco de acción consensuado sobre la aceptación o no de los riesgos derivados de la tecnología objeto de la misma? ¿Por qué remitir esta cuestión en exclusiva al modelo regulatorio clásico, que ya ha mostrado sus limitaciones y fatiga en este caso (energía nuclear, ingeniería genética, clonación o nanotecnología, por ejemplo)? La visión más clásica del modelo se justificará en que ello responde a criterios de seguridad e interés público respecto de las consecuencias del uso de las tecnologías, obviando una 
premisa insoslayable: ¿dónde subyace el consenso para justificar el uso o la prohibición de una tecnología en concreto? No parece que los parlamentos y sus lobbies constituyan el marco más acreditado para justificar por sí solos la comprensión pública y consenso social respecto de una tecnología en concreto, sino que más bien responden a un mecanismo asimétrico del uso de la información.

Así pues, la regulabilidad basada en la regulación clásica debe ser necesariamente complementada con mecanismos autorregulatorios que a su vez pueden incorporar, ya sea en la norma técnica misma o en momentos posteriores, procesos de gobernanza del riesgo que contribuyan a una más transparente y distribuida aceptación de responsabilidad basada en la gestión de intereses de los stakeholders sobre la base de un uso simétrico de la información.

Es así como los conceptos de autorregulación y gobernanza del riesgo pueden confluir para facilitar la comprensión pública de la tecnología y la aceptación o no de los riesgos construidos a partir de su introducción en "lo" social: incorporando, de un lado, la autorregulación a los procesos de gobernanza del riesgo; y, del otro, la gobernanza del riesgo a los corpus normativos autorregulados.

\section{UN EJEMPLO: EL CASO DE LAS NANOTECNOLOGÍAS}

Las nanotecnologías son vistas como uno de los avances tecnológicos más prometedores del siglo XXI. Consisten en la fabricación, manipulación y control de los materiales a un nivel por debajo de la escala atómica. En muchos aspectos son resultantes de una serie de desarrollos adicionales en física, química y bioquímica, física cuántica, ciencias de los materiales y la metrología. Es así que, en tanto que emergente familia de heterogéneas y revolucionarias tecnologías, es definida por su escala —el nanómetro (nm) - lo que permite la manipulación de la materia a nivel atómico (Drexler, Peterson, y Pergamit, 1993). La materia se comporta de manera distinta en la nanoescala, lo que permite a los investigadores crear estructuras y elementos de una forma que no es posible al nivel molecular tradicional. En la actualidad, una multitud de productos incorporan nanotecnologías con nanoproductos comercialmente disponibles incluyendo composiciones para el uso en alimentos, pesticidas, protectores solares, cosméticos, cámaras digitales, ropa deportiva, etc. (Environmental Law Institute; ETC Group; Pinson, 2004). En contraste con estos simples nanoproductos pasivos, el futuro de las aplicaciones nanotecnológicas promete beneficios sociales significativos, incluyendo las mejoras en los diagnósticos médicos y en los tratamientos de salud, en recursos energéticos más eficientes, materiales más económicos, ligeros y más rápidos (Roco, 2005; Royal Society and Royal Academy of Engineering [RS-RAE], 2004; Wood et al., 2003).

La investigación más "común" del riesgo en el ámbito de las nanotecnologías, como en otras tecnologías emergentes, se ubica en el análisis de la contingencia de un daño - es decir, su anticipación mental, en cuanto a lo que conocemos como dañino, que puede o no producirse, pero que, en cualquier caso, es posible cuantificar-. Como describíamos anteriormente, se trataría en realidad de "peligros". En el ámbito de las tecnologías emergentes, con elevada incertidumbre, no es posible anticipar mentalmente daños pues, precisamente en su "calidad" de emergente, no existe experiencia previa del daño ni es posible medir, prevenir, cuantificar ni, por tanto, asegurar contingencias. Es en este contexto incierto dónde se cultivan los riesgos - esto 
es, constructos sociales, políticos, económicos, religiosos, etc., respecto de situaciones que no podemos prever por cuanto las desconocemos y por tanto difícilmente podemos medir, cuantificar o prevenir-. ${ }^{4}$

Nos ubicamos por tanto ante los riesgos - no los peligros - asociados a las nanotecnologías. Nos ubicamos frente a los tótems que genera en lo social la incertidumbre de la complejidad sociotécnica. Unos tótems que habitan tanto el debate social, la comprensión pública de la ciencia como la gestión de los intereses de los stakeholders implicados. Sirva como ejemplo un informe de 2007 del Economic and the Social Research Council del gobierno inglés, que identificó como uno de los ejes más relevantes del debate sobre las nanotecnologías (ESRC, 2007) el que se fundamenta en las consecuencias sociales y económicas - positivas y negativas- de las nanotecnologías. Esto ha caracterizado buena parte de la literatura que, desde el punto de vista de las ciencias sociales, ha analizado el fenómeno (Kulinowski, 2004; Pense y Cutcliffe, 2007; Selin, 2007), la comprensión pública de la ciencia (Coob y Macoubrie, 2004; Gaskell et al., 2005; Macoubrie, 2006), la sociología del riesgo (Petersen et al., 2007; Powell, 2007; Rogers-Hayden y Pidgeon, 2007), o la comunicación (Pidgeon et al., 2011). El resultado principal de esta discusión ha sido la génesis de un terreno ambiguo caracterizado por el desconocimiento y la incertidumbre con valoraciones (assessments) claramente divergentes y fuertemente polarizadas (Petersen et al., 2007; Powell, 2007). Por otra parte, han existido también visiones optimistas (Bruce, 2005; Salamanca-Buentello et al., 2005) que han defendido las potencialidades de esta nueva tecnología en la resolución de problemas como la pobreza, el hambre en el mundo o el esperanza de vida. U otras que se plantean visiones más pesimistas (Dunkley, 2004) que, estableciendo analogías con el caso de los organismos genéticamente modificados, han sugerido escenarios ejemplares de los efectos disruptivos que las nanotecnologías podrían tener en la sociedad. En el contexto de esta distinción las ciencias sociales, pese a ser un agente potencial de desarrollo tanto en la investigación en nanotecnologías como en sus procesos de innovación (Macnaghten et al., 2005) han tendido a priorizar el estudio del compromiso público en el desarrollo tecnológico por encima del estudio de la representación y de la construcción social de los riesgos en este ámbito.

Sin duda, los cambios sociales que se derivan de la revolución tecnológica sitúan los instrumentos que la hacen posible en el centro mismo de la vida de las personas. El caso de las nanotecnologías es un ejemplo claro. La emergencia de los cambios inauguran nuevas formas de interacción entre la tecnología y la sociedad, fenómeno históricamente no exento de polémicas. En el marco de un contexto de naturaleza marcadamente sociotécnica - es decir, de una estructura social constituida, por un lado, por artefactos técnicos y, por otro, por el conjunto de complejas relaciones que se establecen entre los diferentes actores sociales-, la sociedad contemporánea se ve enfrentada a un volumen creciente de cuestiones relativas a la complejidad científica y tecnológica y, con ellas, a la controversia social generada por la introducción de las nuevas tecnologías. Fruto de las dinámicas cambiantes que originan las tecnologías en la sociedad así como las respuestas sociales hacia ellas, se erigen nuevos frentes que demandan ser atendidos mediante el uso de herramientas y de procedimientos que se adecuen a la realidad, a la realidad multidisciplinar y multidimensional que ca-

\footnotetext{
Cfr. Supra.
} 
racteriza la realidad sociotécnica de nuestras sociedades, de ahí nuestra apuesta por la "gobernanza anticipatoria".

En efecto, la complejidad de gran parte de los sistemas técnicos reside, no sólo en su carácter meramente tecnológico, sino también en la resolución de determinadas dinámicas sociales que se originan como consecuencia de la emergencia de aquellos. Una aproximación científica de carácter social a las nanotecnologías debería revelar por una parte, la manera cómo los diferentes actores colonizan el futuro, y cuestionar, por otro, las formas de verdad y de legitimidad en el entorno del futuro mismo. En este marco, saber quién tiene legitimidad y conocer el tipo de métodos que sostienen dicha legitimidad se convierte en una cuestión crítica y con un peso directo en la relación entre el presente y el futuro (Petersen et al., 2007). El desarrollo del dominio tecnológico es, a menudo, atribuido a varios factores, entre ellos, la convergencia de disciplinas, la expansión de instituciones así como otros aspectos de la vida política, económica, social y cultural. Tanto estos elementos como las interacciones y las negociaciones entre los actores pueden afectar la forma en que el campo de conocimiento es constituido y reconstituido, así como sus posibilidades y expectativas. Esto nos puede conducir a afirmar que, en tanto que abstracción temporal (Selin, 2007: 197), el futuro de las nanotecnologías es construido y gestionado socialmente de manera permanente, y con ello resulta importante conocer por parte de quien "emerge" y se "producen" las nanotecnologías y bajo qué condiciones se construye y se difunde. Si la sociedad acepta, aprovecha y utiliza los hallazgos de una tecnología entonces ésta sobrevive, si no lo hace, entonces no importa cuánto mejor sea esa tecnología pues ella fracasará.

La percepción y el conocimiento son partes importantes de la comprensión pública de la ciencia. Contrariamente a lo que preocupa a los científicos, los miedos públicos sobre los riesgos tecnológicos son riesgos mucho menos atribuibles a la tecnología que al contexto social y regulatorio en el que aquellos se adhieren. Es por este motivo que algunos autores advierten que una mala comprensión de las preocupaciones públicas está conduciendo a incrementar la desafección pública e incluso, a una menor confianza hacia los científicos y los cuerpos regulatorios. La confianza juega pues un rol muy importante en el fenómeno de la opinión pública sobre la nanotecnología.

Los investigadores en ciencias sociales y naturales están sólo ahora empezando a ver la importancia de desarrollar una comprensión rigurosa sobre la formación de la opinión pública en torno a las nanotecnologías. De hecho, muchos de estos primeros estudios se centraron principalmente en un análisis descriptivo de la cobertura de los medios (Gaskell et al., 2005) o sobre la percepción de los riesgos y los beneficios de la nanotecnología (Bainbridge y Roco 2005; Cobb y Macoubrie, 2004). ${ }^{5}$

\footnotetext{
5 Durante los años 2004 y 2005 se desarrollaron dos investigaciones sobre el conocimiento y las actitudes públicas sobre la nanotecnología. Los resultados de estos dos grandes estudios señalaron que muy pocas personas en los EEUU sabían algo sobre nanotecnología en ese momento. También se concluyó que aquellos que eran más conocedores de la nanotecnología tenían actitudes más positivas y con más expectativas hacia los beneficios que los riesgos. Este tipo de interpretación puede conducir a pensar que cuando las personas están más familiarizadas con las nanotecnologías, más las aceptarán. Ésta es la hipótesis llamada "familiaridad". Asimismo, cuanta mayor información sea diseminada, su financiación resultará políticamente más aceptable. Sin embargo, estos mismos resultados se pueden conducir a un significado diferente: cuando las personas consideran atractiva la nanotecnología (habiendo leído, por ejemplo, Engines of creation de Eric Drexler), buscan información adicional. Si bien, podríamos pensar que están informados porque son partidarios, en lugar de ser partidarios porque están informados.
} 
En cualquier caso, el análisis del proceso de inserción social de las nanotecnologías nos lleva a identificar varios stakeholders relevantes: los científicos, los empresarios, los reguladores, los medios de comunicación y los ciudadanos. Los científicos se mueven en el ámbito de la verificación de las hipótesis de trabajo (verdadero / falso), los empresarios en el ámbito de la rentabilidad de las inversiones (pérdida / ganancia), los reguladores en el ámbito de las autorizaciones y prohibiciones (subvención / sanción), los medios de comunicación en lo mediático (relevante / irrelevante) y los ciudadanos en la aceptación-uso/rechazo-no uso. Para poder inserir una tecnología en lo social es preciso localizar y gestionar puntos de encuentro de los diversos stakeholders que permitan gobernar el riesgo. En otras palabras desarrollar un proceso de gobernanza anticipatoria del riesgo.

\section{A MODO DE CONCLUSIONES}

Sintetizando los elementos expuestos, podemos concluir que la gobernanza de riesgos sociotécnicos vinculado a las tecnologías emergentes se debate entre la incertidumbre científica y la complejidad inherente de la construcción social de los mismos. La gestión de estos riesgos en el ámbito de la prospectiva y la inteligencia competitiva conlleva importantes efectos en la medida en que la gobernanza de riesgos en el ámbito de ciertas tecnologías presenta dificultades tales que conducen al bloqueo del despliegue de aquellas.

La ausencia de evidencia científica sobre los riesgos asociados a algunas de estas tecnologías no es argumento suficiente para la modificación de la percepción del riesgo existente basada en una construcción social que sólo puede ser abordada por una perspectiva transdiciplinar acorde con la naturaleza de este fenómeno que requiere, por demás, de mecanismos de amplia participación social en la gestión de la incertidumbre. La apuesta de la gobernanza anticipatoria en su vertiente práctica, podría ayudar a resolver dicha situación.

En este sentido el riesgo debería asumirse como un concepto de carácter integral, de voluntad integradora e innovadora, que huya del reduccionismo y contribuya a una perspectiva amplia del entorno socio técnico en que el concepto se ubica. Para ello las ciencias sociales, en un enfoque transdiciplinar, permiten disponer de un marco general en el que interconectar las distintas perspectivas. Ello es, obviamente, predicable también de los riesgos asociados a las tecnologías emergentes, cuya gestión habrá de pasar de un enfoque basado en el cálculo de probabilidades negativas a uno en que la participación de los distintos stakeholders (sector público, sector privado y sociedad civil) complemente los clásicos análisis políticos en el diseño y cuestionamiento de alternativas tecnológicas.

La gestión regulatoria de la gobernanza del riesgo en cuanto a legitimación, representación, procedimiento y expresión de intereses se ve lastrada por los modelos regulatorios clásicos que, al ser indispensables, imposibilitan la negociación de elementos esenciales de la gestión del consenso. De otro lado, los recursos autorregulatorios de base privada y voluntaria, sí que permiten abordar estos aspectos en aras de un mejor desarrollo de los recursos procedimentales de gobernanza de riesgos. 


\section{REFERENCIAS}

Bainbridge, William S.; Roco, M. (2005) Managing nano-bio-info-cogno innovations: Converging technologies in society. Springer: The Netherlands. <http://www.wtec.org/ ConvergingTechnologies/3/NBIC3_report.pdf> (último acceso abril 2013).

Burgess, A. (2002) "Comparing responses to perceived health risks from mobile phone masts". Health, Risk \& Society, 4(2): 175-188. <http://www.sante.gouv.fr/IMG/pdf/ Burgess.pdf> (último acceso enero 2013).

Cobb, MD.; Macoubrie, J. (2004) “Public perceptions about nanotechnology: Risks, benefits and trust". Journal of Nanoparticle Research, 6 (4): 395-405. <http://cms.springerprofessional.de/journals/JOU=11051/VOL=2004.6/ISU=4/ART=5383394/BodyRef/PDF/11051_2004_Article_5383394.pdf> (último acceso abril 2013).

Drexler Eric K.; Peterson, Ch.; Pergamit, G. (1993) Unbounding the future: Nanotechnology revolution. Berkshire: United Kingdom.

Dunkley, RWS (2004) "Nanotechnology: social consequences and future implications”. Futures, 36: 1129-1132.

European Research Council (2007) Nanotecnology the science to: From the social. <http:// www.esrc.ac.uk/_images/Nanotechnology_science_to_social_tcm8-13537.pdf> (último acceso febrero 2013).

Fisher E.; R.L. Mahajan; C. Mitcham (2006) “Midstream modulation of technology: Governance from within”. Bulletin of Science, Technology and Society. 26(6): 485-496. <http:// sciencepolicy.colorado.edu/admin/publication_files/resource-2482-2006.16.pdf> (último acceso marzo 2013).

Funtowicz, S. O. and Ravetz, J. R. (1992) “The emergence of post-normal science”, en von Schomberg, R. (ed.), Science, politics and morality. Kluwer Academic Publishers, Dordrecht: 85-123.

Garcia Hom, A. (2005) Negociar el riesgo. Barcelona: Ariel. (2012) "Aprendiendo del futuro: Gobernando la nanotecnología". Revista CTS, 7: 261-272. <http://www.revistacts. net/index.php?option=com_content $\&$ view=article\&id=461:3-aprendiendo-del-futuro-gobernando-la-nanotecnologia\&catid=110:dossier\&Itemid=98> (último acceso abril 2013).

Gaskell, G.; Ten Eyck, T.; Jackson, J.; Veltri, G. (2005) "Imagining nanotechnology: Cultural support for technological innovation in Europe and the United States". Public Understanding of Science, 14: 81-90.Disponible en: <http://peer.ccsd.cnrs.fr/ docs/00/57/10/57/PDF/PEER_stage2_10.1177\%252F0963662505048949.pdf> (último acceso marzo 2013).

Hutter, B. (2007) “Risk, regulation and management”, en P. Taylor-Gooby y J. O. Zinn (eds.) Risk in Social Science. Londres: Oxford University Press: 202-227.

Krimsky, S.; Golding, D. (eds.) (1996) Social theories of risk. Praeger Paperback.

Kulinowski, K. (2004) "Nanotechnology: From 'wow' to 'yuck'?". Bulletin of Science Technology Society, 24 (1): 13-20. <http://bst.sagepub.com/content/24/1/13.full. pdf + html $>$ (último acceso abril 2013)

Lessig, L. (2000) “Code and other laws of cyberspace”. <http://www.code-is-law.org/> (último acceso marzo 2013).

Macnaghten, P.; Kearnes, MB.; Wynne, B. (2005) "Nanotechnology, governance, and public deliberation: What role for the social sciences?" Science Communication, 27 (2): 
268-291. <http://csec.lancs.ac.uk/docs/nano\%20project\%20sci\%20com\%20proofs\%20nov05.pdf> (último acceso abril 2013).

Macoubrie, J. (2006) “Nanotechnology: Public concerns, reasoning and trust in government". Public Understanding of Science, 15 (2): 221-241. <http://pus.sagepub.com/ content/15/2/221.full.pdf+html> (último acceso marzo 2013).

Park, R. (2000) Voodoo science. New York: Oxford University Press.

Pense, CM.; Cutcliffe, SH. (2007) "Risky talk: Framing the analysis of the social implications of nanotechnology". Bulletin of Science Technology Society, 27 (5): 349-366. <http:// bst.sagepub.com/content/27/5/349.full.pdf> (ultimo acceso marzo 2013).

Petersen, A.; Anderson, A.; Wilkinson, C.; Allan, S. (2007) “Editorial: Nanotechnologies, risk and society". Health, Risk \& Society, 9 (2): 117-124.

Pidgeon, N.; Harthorn, B.; Satterfield, T. (2011) "Nanotecnologies risk perceptions and communication: Emerging technologies, emerging challenges". Risk Analysis, 31(11): 1694-1700.

Pinson, Robert D. (2004) "Is nanotechnology prohibited by the biological and chemical weapons conventions”. Berkeley Journal International Law, 22, 279-309. <http:// scholarship.law.berkeley.edu/bjil/vol22/iss2/4> (último acceso abril 2013).

Powell, MC. (2007) “New risk or old risk, high risk or no risk? How scientists' standpoints shape their nanotechnology risk frames". Health, Risk \& Society, 9 (2): 173-190.

Renn, O.; Klinke, A. (2001) Environmental risk-perception, evaluation and management: Epilogue, en Gisela Böhm, Josef Nerb, Timothy McDaniels y Hans Spada (eds.), Environmental risks: Perception, evaluation and management. Amsterdam: Elsevier Science: 275-299.

Roco, M. (2005) "International perspective on government nanotechnology funding in 2005". Journal of Nanoparticle Research, vol. 7(6): 707-712. <http://www.nsf.gov/ crssprgm/nano/reports/mcr_05-0526_intpersp_nano.pdf> (último acceso marzo 1013).

Rogers-Hayden, T.; Pidgeon, N. (2007) “Moving engagement "upstream”? Nanotechnologies and the Royal Society and Royal Academy of Engineering's inquiry". Public Understanding of Science, 16 (3): 345-364. <http://pus.sagepub.com/content/16/3/345. full.pdf+html $>$ (ultimo acceso marzo 1013).

Royal Society and Royal Academy of Engineering (2004) Nanoscience and nanotechnologies: Opportunities and uncertainties. <http://www.nanotec.org.uk/finalReport. htm> (último acceso marzo 1013).

Salamanca-Buentello, F.; Persad, DL.; Court, EB.; Martin, DK.; Daar, AS.; Singer, PA. (2005) "Nanotechnology and the developing world". PloS Medicine, 2(4): 300-303.

Selin, C. (2007) "Expectations and the emergence of nanotechnology". Science Technology Human Values, 32 (2): 196-220. <http://sth.sagepub.com/content/32/2/196.full. pdf+html> (último acceso marzo 1013).

Stilgoe, J. (2007) "The (co-)production of public uncertainty: UK scientific advice on mobile phone risks". Public Understanding of Science, 16: 45-61. <http://pus.sagepub.com/ content/16/1/45.full.pdf+html> (último acceso marzo 1013).

Szerszynski, B.; Lash, S.; Wynne, B. (1996) “Introduction: Ecology, realism and the Social Sciences”, en B. Szerszynski y S. Lash y B. Wynne (eds.) Risk, environment \& Modernity. Londres: SAGE, 1-26. 
Mundo Nano | Artículos | Vol. 6, No. 10, enero-junio, 2013 | www.mundonano.unam.mx

Wynne, B. (1992) "Misunderstood misunderstandings: Social identities and public uptake of science". Public Understanding of Science, 1: 281-304.

Wood, S.; Jones, R.; Geldart, A. (2003) The social and economic challenges of nanotechnology. Economic and Social Research Council.

\section{Webgrafía}

ETC Group <http://www.etcgroup.org> (último acceso abril 2013).

The Royal Society <http://www.nanotec.org.uk> (último acceso enero 2013).

Environmental Law Institute <http://www.eli.org> (último acceso enero 2013). 